\title{
Gamma correction FCM algorithm with conditional spatial information for image segmentation
}

\author{
Yang Liu ${ }^{1,2}$, Haipeng Chen ${ }^{1,2}$, Xuanjing Shen ${ }^{1,2}$ and Yongping Huang ${ }^{1,2^{*}}$ \\ ${ }^{1}$ College of Computer Science and Technology, Jilin University, Changchun, China \\ [e-mail: liu_yang16@mails.jlu.edu.cn] \\ ${ }^{2}$ Key Laboratory of Symbolic Computation and Knowledge Engineering of Ministry of Education, Jilin University, \\ Changchun, China \\ [e-mail: hyp@jju.edu.cn] \\ *Corresponding author: Yongping Huang
}

Received January 1, 2018; revised February 6, 2018; accepted April 2, 2018; published September 30, 2018

\begin{abstract}
Fuzzy C-means (FCM) algorithm is a most usually technique for medical image segmentation. But conventional FCM fails to perform well enough on magnetic resonance imaging (MRI) data with the noise and intensity inhomogeneity (IIH). In the paper, we propose a Gamma correction conditional FCM algorithm with spatial information (GcsFCM) to solve this problem. Firstly, the pre-processing, Gamma correction, is introduced to enhance the details of images. Secondly, the spatial information is introduced to reduce the effect of noise. Then we introduce the effective neighborhood mechanism into the local space information to improve the robustness for the noise and inhomogeneity. And the mechanism describes the degree of participation in generating local membership values and building clusters. Finally, the adjustment mechanism and the spatial information are combined into the weighted membership function. Experimental results on four image volumes with noise and IIH indicate that the proposed GcsFCM algorithm is more effective and robust to noise and IIH than the FCM, sFCM and csFCM algorithms.
\end{abstract}

Keywords: Image segmentation, MRI brain images, FCM, GcsFCM 


\section{Introduction}

The main purpose of image segmentation is to split the image into the homogeneous connected areas of internal consistency according to the similar characteristics (such as gray, color and texture). Thereby the region of interest (ROI) is extracted from the complex background area for target recognition. However, manual segmentation often depends on the Doctor's knowledge and experience, which increases the possibility of errors. The defects in brain MR images, such as noise, intensity inhomogeneity(IIH), partial volume effect of brain tissue and low contrast, make the MRI brain image segmentation process more challenging. Therefore, computer-assisted segmentation is critical. In the past, a number of medical image segmentation algorithms based on threshold, region, edge and clustering have been proposed. Among them, the clustering segmentations are most frequently used [1-4].

In the clustering segmentation methods, the pixels in the image space are clustered in the corresponding feature space, then they are mapped back to the original image space to obtain split results. And FCM is commonly used among the clustering algorithms [5-8]. It has fuzzy nature that one pixel can belong to multiple clusters, which can lead to better performance than hard clustering methods. The unsupervised FCM algorithm can reduce the human intervention, which is more suitable for the images with the characteristics of uncertainty and fuzziness. But it is too sensitive to the initial parameters, and sometimes requires the initialization of the artificial intervention parameters to approach the global optimal solution and improve the segmentation speed. In addition, the traditional FCM algorithm does not take into account the spatial information, so that it is very sensitive to noise and IIH. To solve these problems, a lot of improved fuzzy clustering algorithms have been proposed.

In order to overcome the effect of noise, Yang et al. [9] proposed the penalized FCM (PFCM) algorithm that introduced the neighborhood information penalty term in the objective function of FCM. It effectively reduces the influence of noise in the segmentation process, but it has no effect on the IIH. Chen and Zhang [10] used the mean filtering and median filtering in FCM, respectively. Based on FCM with mean filtering, Szilagyi et al. [11] introduced the grayscale histogram to achieve the rapid image segmentation. However, either median filtering or mean filtering inevitably blurs edge information of the image, which results in decreasing the segmentation accuracy. Chuang et al. [12] used spatial information to update the membership function twice in the FCM algorithm because the neighborhood pixel had the same attributes as the central pixel. The objective function did not change in space FCM (sFCM), which has advantages for the noise. Ji et al. [13] introduced a new approach to use the spatial information in FCM. In this method, image patches are considered instead of just pixels, which add additional information to the data. The noise is handled effectively by utilizing the spatial information, but it increases computational burden. Bai et al. [14] introduced the nonlocal spatial information and used the spatial shape information of the contour of the image to refine the local spatial constraint by Markov random field. It is effective to the noise. To reduce the effect of IIH, Ahmed et al. [15] proposed a biased field estimation FCM(BCFCM) algorithm, which achieves the purpose of compensating the intensity inhomogeneity by introducing the neighborhood normalization term to modify the objective function. Although this regularization is useful in segmenting images with salt and pepper noise, it introduces additional complexity.

To solve the effect of noise and IIH, Ji et al. [16] proposed a robust spatially constrained fuzzy c-means (RSCFCM) algorithm. A novel factor is introduced by considering the spatial 
direction, which is calculated based on the posterior and prior probabilities. The algorithm can substantially improve the accuracy of brain MR image segmentation. Adhikari et al. [17] proposed the conditional spatial fuzzy C-means (csFCM) algorithm. They constructed the space constraint regular term which merged into the local space information by the membership function. And the regular term describes the degree of participation of each pixel in constructing membership function, which can effectively segment the MRI brain image with noise and IIH.

For many clinical applications, brain images need to be divided into different tissue types. However, Jiang et al. [18] proposed a local correntropy space constrained FCM (LCFCM_S) and its simplified model (LCFCM_S1). The correntropy standard can effectively enhance the weight of the sample near its clustering center. The algorithm can handle images with noise, low contrast and IIH, but it cannot segment images with different tissue types.

In conclusion, most of the above-mentioned algorithms are not robust for the segmentation performance of images with high percentage noise and IIH. And the brain images which often accompany with narrow topological structures are complex. Some methods cannot segment brain images with different tissue types. In addition, the algorithms cannot make good use of the neighborhood information. The classification may be often overlooked the details of the image, causing many isolated spots and the image edges blurred.

To address these problems, we propose a novel Gamma correction conditional space FCM(GcsFCM) algorithm in this paper. Firstly, the Gamma correction is used to highlight the detail information of the noise and IIH images. By enhancing the contrast of the images, we can effectively deal with blurring of image boundaries due to noise and IIH. And considering the different importance of the target neighborhood pixels, neighborhood effective mechanism as an adjustment factor is introduced. Secondly, we introduce it into the local spatial information. Finally, the local and global spatial information are integrated into the weighted membership function. The experiments show that GcsFCM algorithm can effectively segment MRI brain images with the presence of noise and IIH. In addition, we have also made some comparisons with several state-of-the-art models to show the superiority of our method.

The remainder of this paper is organized as follows: Section 2 briefly introduces the FCM algorithm. Section 3 states the detail of the proposed GcsFCM algorithm. Section 4 describes the results and discussion of algorithms for qualitative and quantitative assessment of MRI. Finally, Section 5 gives a summary and some conclusions.

\section{FCM algorithm}

FCM algorithm, based on the objective function, is a constrained nonlinear programming problem [5-8]. It uses the fuzzy membership degree $U$ and non-similar measure $d$ (usually using Euclidean distance) to construct the objective function and sets the termination condition to achieve the fuzzy segmentation of the image. For the image $X=\left\{x_{1}, x_{2}, \ldots, x_{N}\right\}$ to be segmented, the membership function $p_{i k}$ and the clustering center $v_{i}$ are updated by minimizing the objective function, which can be expressed as:

$$
\begin{aligned}
J_{F C M}(U, V) & =\sum_{i=1}^{C} \sum_{k=1}^{N} p_{i k}^{m} d_{i k}^{2} \\
& =\sum_{i=1}^{C} \sum_{k=1}^{N} p_{i k}^{m}\left\|x_{k}-v_{i}\right\|^{2}
\end{aligned}
$$


where $N$ is the number of pixels, $C$ is the number of clusters, $m$ is the fuzzy factor, $v_{i}$ is the clustering center of the $i^{\text {th }}$ cluster, and $p_{i k}$ is the fuzzy membership of the pixel $x_{k}$ belonging to the $i^{\text {th }}$ cluster. And it satisfies the constraint:

$$
\sum_{i=1}^{C} p_{i k}=1,0 \leq p_{i k}<1, k=1,2, \ldots, N
$$

The loss function in Eq. (1) can be minimized by lagrangian multiplier method. And the membership function and the clustering center are updated as follows:

$$
p_{i k}=\frac{1}{\sum_{i=1}^{C}\left(\frac{\left\|x_{k}-v_{i}\right\|}{\left\|x_{k}-v_{c}\right\|}\right)^{\frac{2}{m-1}}}
$$

and

$$
v_{i}=\frac{\sum_{k=1}^{N} p_{i k}^{m} x_{k}}{\sum_{k=1}^{N} p_{i k}^{m}}
$$

We summarize the FCM algorithm as pseudocode snippet, which is shown in Table 1.

Table 1. An image segmentation of FCM scheme pseudocode

1. Input: Image-I

2. Output: Segmented Image-class II

3. Acquire: the values of the number of clusters $C$, the degree of fuzziness $m$, the error $e$ and the value of objective function $J^{(0)}$.

4. Initialize randomly the centres of clusters $v_{i}^{(0)}$

5. $\mathrm{l}=0$

6. Begin:

7. $l=l+1$

8. Calculate the membership degrees $p_{i j}^{(l)}$ using Eq. (3)

9. Update the centers of classes $v_{i}^{(l)}$ using Eq. (4)

10. Calculate the value of objective function $J$ using Eq. (1)
11. If $\left|J^{l}-J^{(l-1)}\right|<e$ Then
Break

\section{End if}

12. End 


\section{The proposed GcsFCM algorithm}

Image segmentation divides the image into different areas according to the pixel grayscale feature [19][20]. In the MRI images, especially with a lot of noise and intensity inhomogeneity, some details of the image would be lost and the information of tissue edges would be blurred. Therefore, the Gamma correction is firstly introduced in this study, which can obviously enhance the medical images of the detail and edge information. And it has advantage of highlighting the image contrast, which can improve the segmentation accuracy.

Just considering the pixel gray information is not enough. And the likelihood that the neighboring pixels and center pixel in the brain image belong to the same class is very large. We have introduced local space information in FCM algorithm. Moreover, the influence of the different pixels to the fuzzy membership degree of the target pixel must be taken into account. Therefore, an effective neighborhood mechanism is introduced to preserve connectivity of the same class and boundary information of different categories. The flow chart of the proposed GcsFCM is shown in Fig. 1. For the formulas involved in the figure, the calculation method is given in detail in sections 3.1 and 3.2.

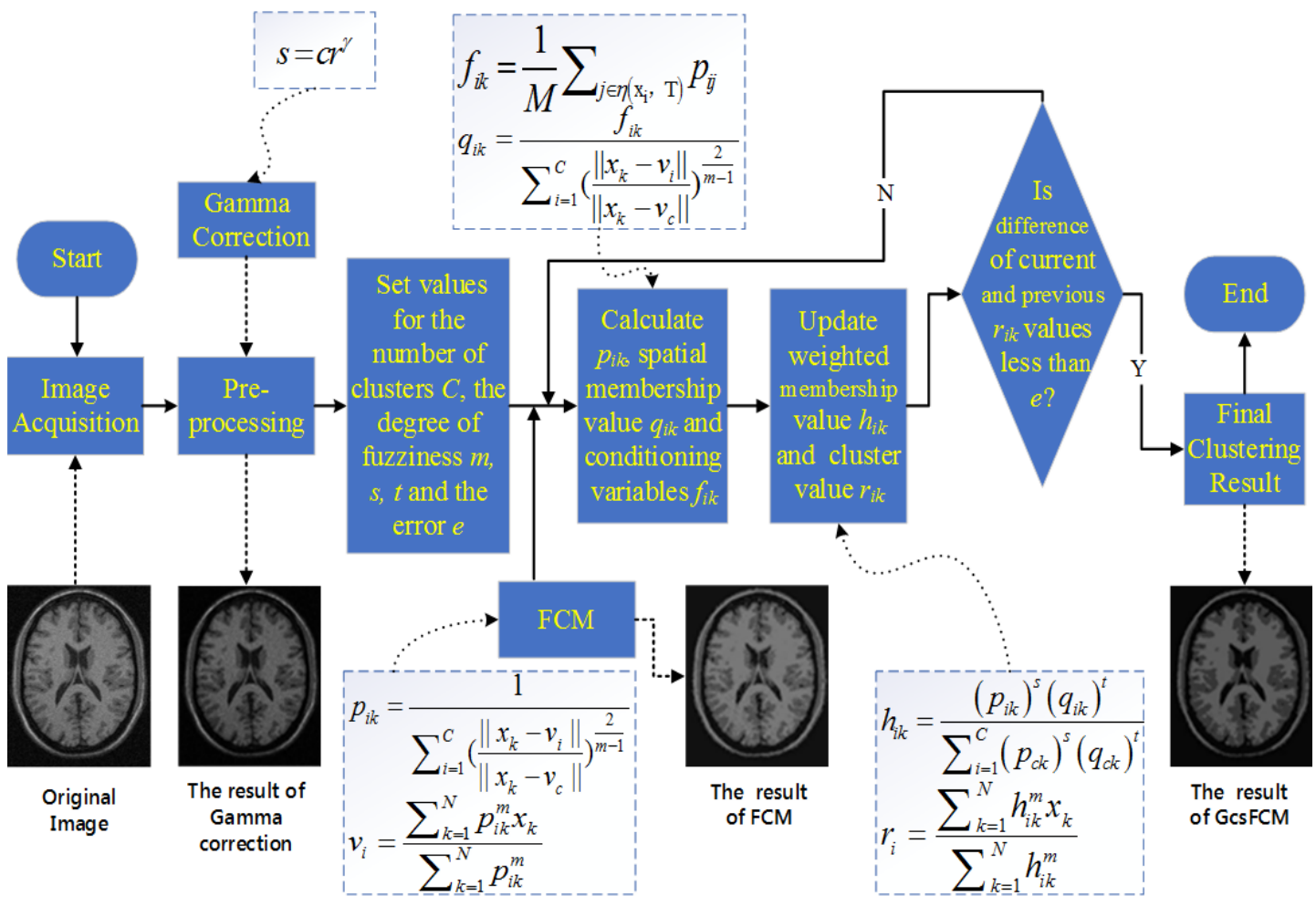

Fig. 1. The flow chart of GcsFCM algorithm. The acquired image is preprocessed by Gamma correction and then segmented by the conditional spatial FCM algorithm.

\subsection{The pre-processing of gamma correction}

Recently, researchers have proposed many image enhancement methods [21][22][23], such as histogram equalization method [24][25], gray scale linear transformation [26][27] and smoothing and sharpening [28]. The histogram equalization method can improve the image quality, but it often brings some discontinuous patches into the image. The linear 
transformation is not complicated, but it cannot improve the accuracy of the image segmentation. The preprocessing of smoothing and sharpening can make the details of the image loss or excessively highlight the noise information, which affects the final experimental results. In contrast, Gamma correction which detects the dark part and light-colored part in the image signal and edits the image's Gamma curve is a pixel-based nonlinear transformation, hence the image contrast is enhanced. Gamma correction will not bring the discontinuity of the plaque, which does not need to consider the problem of gray inhomogeneity. The image pixel value is corrected to show some advantages in image enhancement [29]. So we preprocess the image by Gamma enhancement to enhance the contrast of the image and further improve the experimental results accuracy. The basic form of the Gamma transformation is:

$$
S=c X^{\gamma}
$$

where $c$ and $r$ are positive constants. Fig. 2 shows the results after Gamma correction. Fig. 2(a) presents the original images of slice 100 and 90. Fig. 2(b) displays the results after Gamma correction.

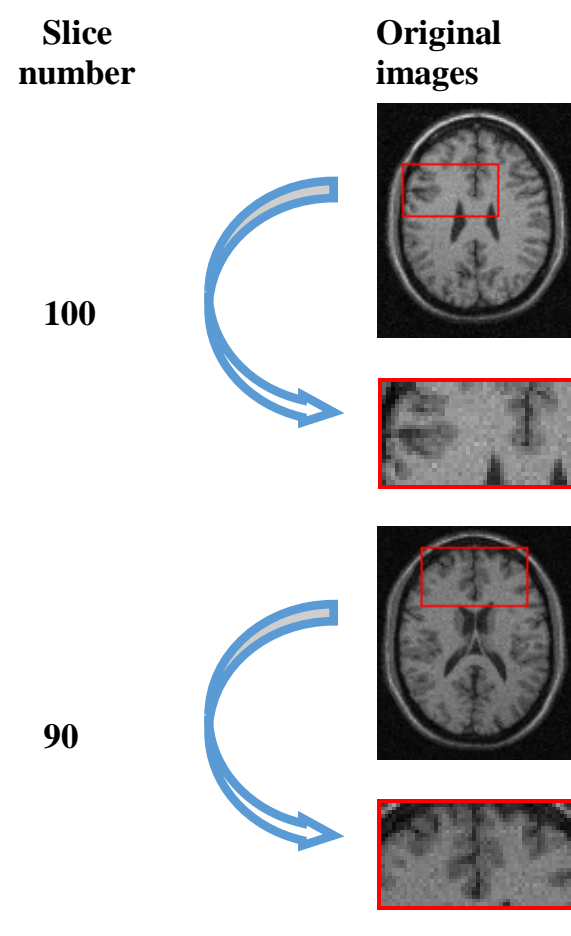

(a)
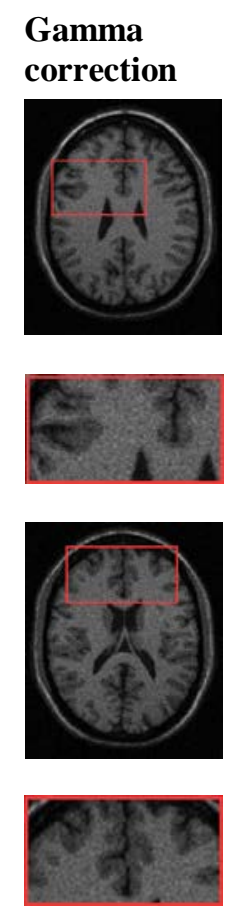

(b)

Fig. 2. The experimental results after pre-processing

Fig. 2 shows the qualitative results after the Gamma correction of T1-weighted images (slice 100 and 90 ) with $9 \%$ noise and $40 \%$ inhomogeneity. In order to better present the experimental results, we take the red area as an example to give a larger image. It can be seen that the contrast of the image has been significantly enhanced and the information of edges and details has become clear. 


\subsection{The effective neighborhood spatial FCM algorithm}

We have incorporated local spatial interaction among adjacent pixels in the fuzzy membership function. It is helpful to reduce the effect of noise and IIH in the segmentation results of MRI images. We also introduce the effective neighborhood mechanism, assuming a corresponding effective neighborhood function value $f_{1}, f_{2}, \ldots, f_{N}$ for all the pixels $x_{1}, x_{2}, \ldots, x_{N}$. The $f_{i}(i=1$, $2, \ldots, N$ ) defines the participation degree of the pixel $x_{i}$ in the final constructed clustering membership value, which can improve the classification accuracy. The effective neighborhood function $f$ is constructed as follows.

Considering that $W$ is a movable pixel window, the larger scale will make the edges smooth, resulting in higher pixel classification errors [11][30]. In this study, we choose $W=3 \times 3$, which contains $n$ pixels. $x_{i}$ is the center pixel and the average value of the pixel in the window is:

$$
C\left(x_{i}\right)=\frac{1}{n} \sum_{j=1}^{n} x_{j}
$$

A highly similar pixel area in the neighborhood is defined as:

$$
\eta\left(x_{i}, T\right)=\left\{x_{j} \in W\left\|C\left(x_{i}\right)-C\left(x_{j}\right)\right\| \leq T ; j \neq i\right\}
$$

In Eq. (7), $\eta\left(x_{i}, T\right)$ reflects the neighborhood pixels closing to the window center pixel $x_{i} . T$ is the difference between the value of the neighborhood pixel and center pixel. It can be considered that the pixels in the highly similar pixel region $\eta\left(x_{i}, T\right)$ have a greater influence on the fuzzy membership degree of the central pixel. While the pixels not in the region is approximately considered as having a large difference from the central pixel and less affecting to the center pixel. $M$ is the number of pixels within the region. The effective neighborhood mechanism $f_{i k}$ defines the degree of participation of pixel $x_{k}$ in the $i^{\text {th }}$ clusters and is defined as follows:

$$
\begin{aligned}
& f_{i k}=\frac{1}{M} \sum_{j \in \eta\left(\mathrm{x}_{\mathrm{i}}, \mathrm{T}\right)} p_{i j} \\
& 0 \leq f_{i k} \leq 1
\end{aligned}
$$

Thus, another conditional local space parameter $q_{i k}$ which defines the degree of belonging of pixel $x_{k}$ to the $i^{\text {th }}$ cluster $v_{i}$ is introduced and defined as follows:

$$
q_{i k}=\frac{f_{i k}}{\sum_{i=1}^{C}\left(\frac{\left\|x_{k}-v_{i}\right\|}{\left\|x_{k}-v_{c}\right\|}\right)^{\frac{2}{m-1}}}
$$

Global and local membership values are combined, so we have introduced another weight membership variable $h_{i k}$ and clustering center $r_{i}$.They are defined as follows: 


$$
h_{i k}=\frac{\left(p_{i k}\right)^{s}\left(q_{i k}\right)^{t}}{\sum_{i=1}^{C}\left(p_{c k}\right)^{s}\left(q_{c k}\right)^{t}}
$$

and

$$
r_{i}=\frac{\sum_{k=1}^{N} h_{i k}^{m} X_{k}}{\sum_{k=1}^{N} h_{i k}^{m}}
$$

where $s$ and $t$ are the parameters to control the relative importance of the two membership functions.

A small value in $p_{i k}$ and $q_{i k}$ does not have much effect on the final membership value $h_{i k}$. This mechanism ensures that the likelihood of a normal pixel point to a highly similar neighborhood grouping is increased, making the compactness inside the cluster better. And it will be weakened the effects of noise and inhomogeneity pixels. Thus, it makes the GcsFCM algorithm robust to the image with the noise and IIH. We summarize the proposed scheme as pseudocode snippet, which is shown in Table 2.

Table 2. An image segmentation scheme pseudocode

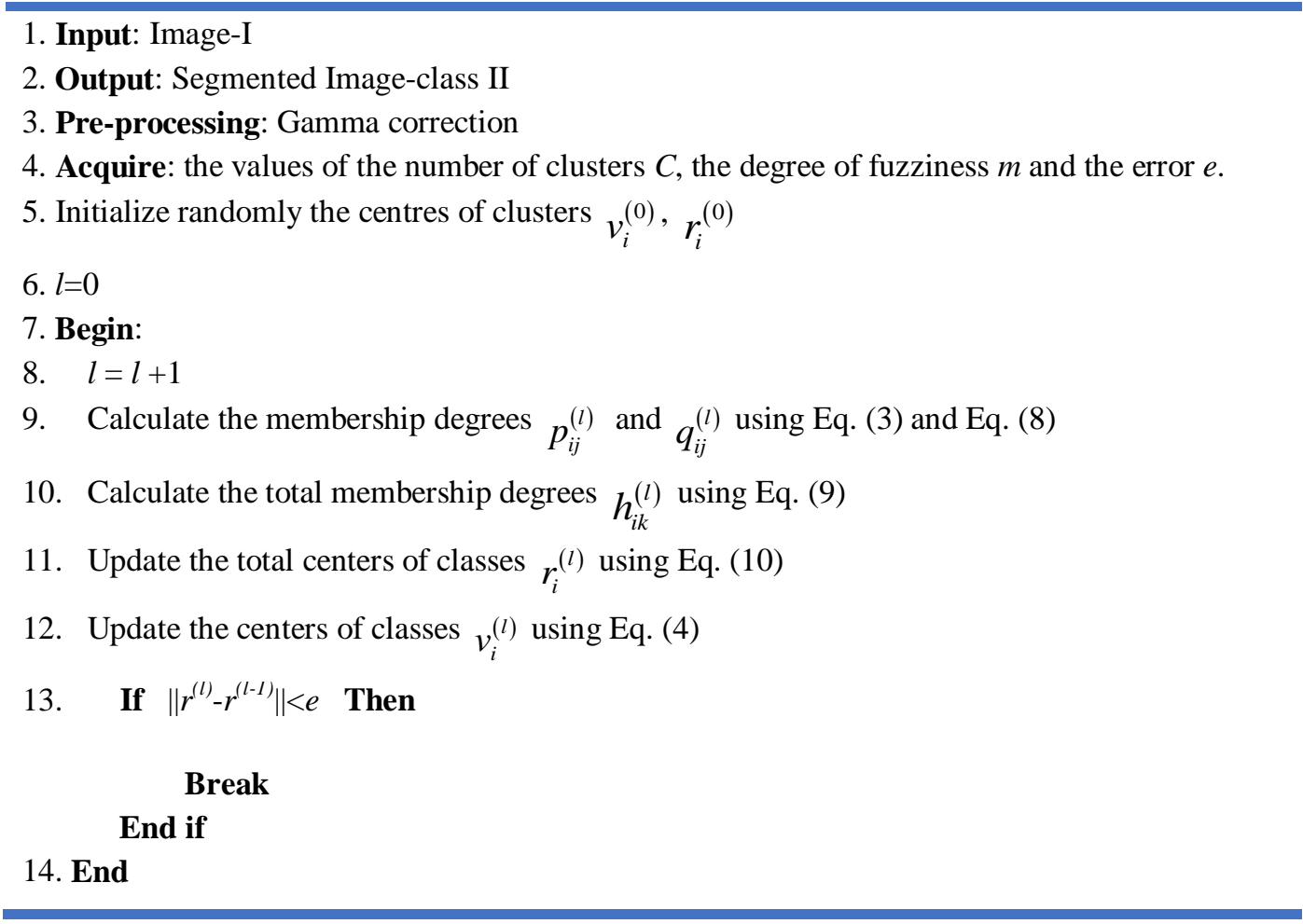

\section{Experimental Results}

The algorithm is experimented and evaluated on BrainWeb simulated MRI brain images [31]. Four different combinations of simulated T1-weighted data volumes which contain images of 9\% noise and 40\% inhomogeneity, 9\% noise and 20\% inhomogeneity, 7\% noise and 40\% 
inhomogeneity, and $7 \%$ noise and $20 \%$ inhomogeneity are selected. Each combination of image volumes consists 71 images. Therefore, the final evaluation results are the average values of 71 images. In the study, we make comparations with the FCM, the sFCM [12] methods as well as the competitive technique, csFCM [17].

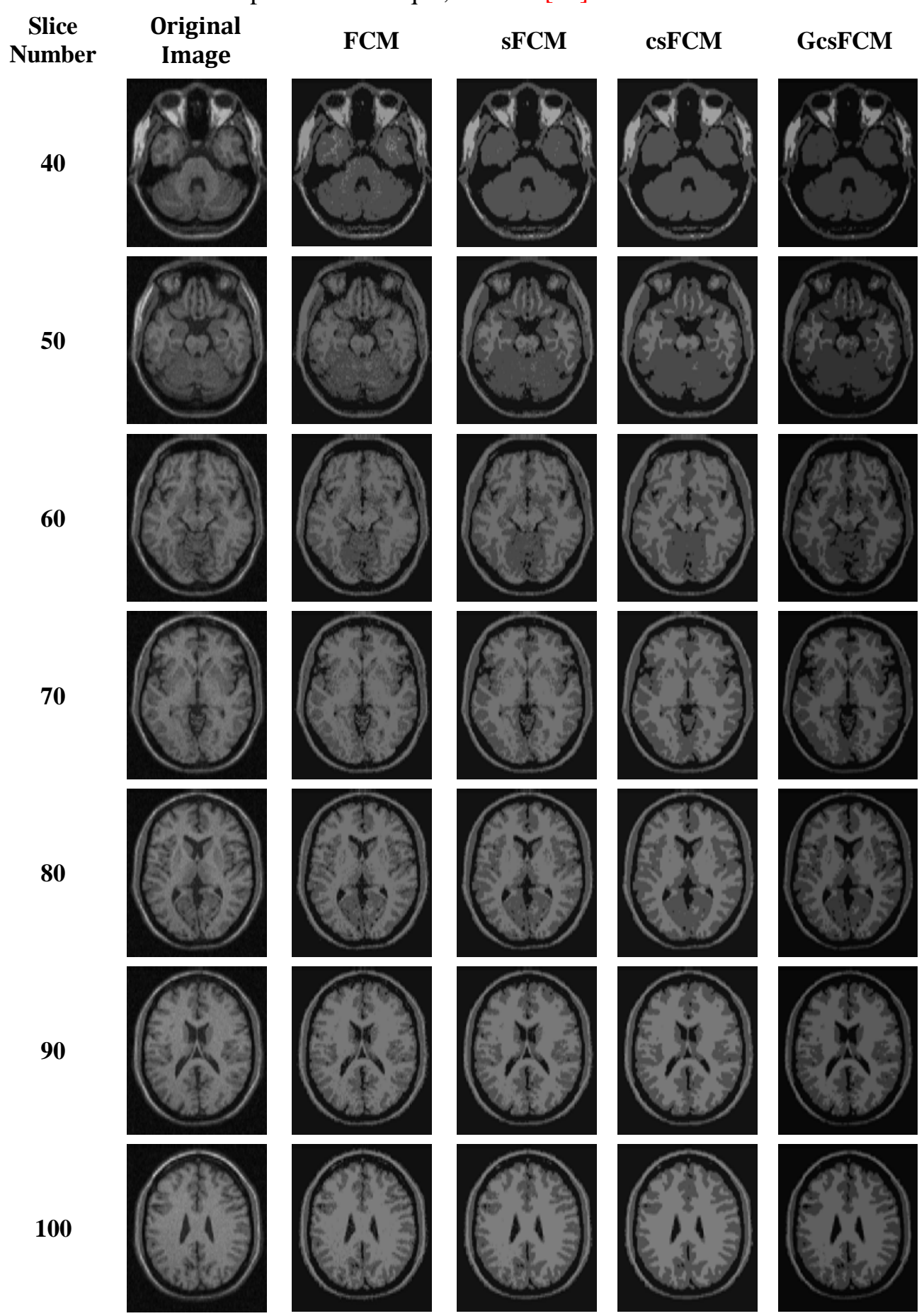

Fig. 3. Comparison results of FCM, sFCM, csFCM and our GcsFCM. Qualitative segmentation of different slice numbers $(40,50, \ldots, 100)$ of T1-weighted MRI brain images with $9 \%$ noise and $40 \%$ inhomogeneity. 


\section{1 parameter setting}

The parameters $p$ and $q$ of the weighted membership function have an important effect on the final membership function value $h_{i k}$ and the clustering center $r_{i}$. According to the empirical value of $p=2$ and $q=2$, the experimental results achieve better performance. In the MRI images, there is a strong correlation between adjacent pixels. It can be noted that the algorithm for $W=3 \times 3$ will get better results [17]. Thus, subsequent experiments were performed by using $p=2, q=2$ and $W=3 \times 3$.

The parameter value of Gamma correction also affects the final segmentation results. A smaller value will make the image edge and detail information more blurred. A larger value will make the noise more prominent. Therefore, it is particularly important to select a suitable value.

\subsection{Qualitative evaluation}

We compare the proposed GcsFCM algorithm with FCM algorithm, sFCM algorithm and csFCM algorithm. In order to avoid the contingency of experimental results, qualitative evaluations include segmentation results in different slice numbers $(40,50, \ldots, 100)$. We have tested the GcsFCM method in T1-weighted simulated of MRI brain image volumes with various levels of noise and inhomogeneity properties.

It should be noted that, for achieving the balance of good readability and appropriate paper length, we show our experimental studies in two parts: the major contents are shown in Section 4 and the others are given in the Appendix as supplementary material.

To perform our experiments completely, we generate four data scenarios suitable for clustering results, as illustrated in Fig. 3 and Fig. A1, Fig. A2 and Fig. A3, respectively. Fig. 3 shows the qualitative results of T1-weighted images with $9 \%$ noise and $40 \%$ inhomogeneity, segmented by FCM, sFCM, csFCM and GcsFCM. In the Appendix, Fig. A1 shows the qualitative results of T1-weighted images with $9 \%$ noise and 20\% inhomogeneity, segmented by FCM, sFCM, csFCM and GcsFCM, Fig. A2 shows the qualitative results of T1-weighted images with $7 \%$ noise and $40 \%$ inhomogeneity, segmented by FCM, sFCM, csFCM and GcsFCM and Fig. A3 shows the qualitative results of T1-weighted images with 7\% noise and $20 \%$ inhomogeneity, segmented by FCM, sFCM, csFCM and GcsFCM.

As it can be seen from Fig. 3, there are several artifacts in the regions of image by FCM and sFCM algorithms. And the image details are affected by the segmentation of csFCM algorithm. The proposed GcsFCM algorithm is basically free of artifacts and isolated points, which retains the details of the image well. Even in the MRI images with high proportion of noise and inhomogeneity, it shows better performance than FCM, sFCM and csFCM algorithms.

\subsection{Quantitative evaluation}

Quantitative evaluation is essential to compare the results of different segmentation methods, which can directly reflect the performance of the algorithm. The validity indices of partition coefficient [14][32][33] and partition entropy [14][33][34] are computed to compare the performance of all algorithms.

(A) Partition coefficient $\left(V_{p c}\right)$ : Partition coefficient $\left(V_{p c}\right)$ is an important indicator of fuzzy partition. The value of $V_{p c}$ is between 0 and 1 . The higher $V_{p c}$ is, the better segmentation effect is. It is defined as follows:

$$
V_{p c}=\frac{\sum_{i=1}^{C} \sum_{k=1}^{N} h_{i k}^{2}}{N}
$$


(B) Partition entropy $\left(V_{p e}\right)$ : Another important indicator of fuzzy partitioning is partition entropy. The value of $V_{p e}$ is between 0 and 1 . The lower $V_{p e}$ is, the better segmentation effect is. It is defined as follows:

$$
V_{p e}=\frac{-\sum_{i=1}^{C} \sum_{k=1}^{N}\left[h_{i k} \log h_{i k}\right]}{N}
$$

Table 3 shows the average of $V_{p c}$ and $V_{p e}$ on FCM, sFCM, csFCM and the proposed GcsFCM algorithms. Each of them is in the four image volumes: $9 \%$ Noise and $40 \%$ IIH, $9 \%$ Noise and 20\% IIH, 7\% Noise and 40\% IIH, and 7\% Noise and 20\% IIH.

Table 3. The values of $V_{p c}$ and $V_{p e}$ on the 71 T1-weighted MRI brain images

Image volume

Noise 9\%, IIH 40\%

\section{Segmented} method

\section{Cluster validity functions}

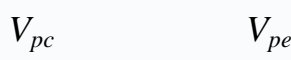

\begin{tabular}{l|l|l} 
FCM & 0.8347 & 0.3033
\end{tabular}

\begin{tabular}{l|l|l} 
SFCM & 0.9580 & 0.0696
\end{tabular}

\begin{tabular}{l|l|l} 
CsFCM & 0.9727 & 0.0449
\end{tabular}

\begin{tabular}{l|l|l} 
GcsFCM & 0.9803 & 0.0326
\end{tabular}

\begin{tabular}{|c|l|l|}
\hline FCM & 0.8348 & 0.3032 \\
\hline sFCM & 0.9585 & 0.0688 \\
\hline CsFCM & 0.9728 & 0.0447 \\
\hline GcsFCM & $\mathbf{0 . 9 8 0 7}$ & $\mathbf{0 . 0 3 2 0}$ \\
\hline
\end{tabular}

Noise 7\%, IIH 40\%

\begin{tabular}{|c|l|l|}
\hline FCM & 0.8385 & 0.2951 \\
\hline sFCM & 0.9599 & 0.0666 \\
\hline CsFCM & 0.9732 & 0.0441 \\
\hline GcsFCM & $\mathbf{0 . 9 8 2 1}$ & $\mathbf{0 . 0 2 9 6}$ \\
\hline
\end{tabular}

Noise 7\%, IIH 20\%

\begin{tabular}{|c|l|l|}
\hline FCM & 0.8397 & 0.2932 \\
\hline sFCM & 0.9610 & 0.0648 \\
\hline CsFCM & 0.9738 & 0.0431 \\
\hline GcsFCM & $\mathbf{0 . 9 8 2 9}$ & $\mathbf{0 . 0 2 8 3}$ \\
\hline
\end{tabular}




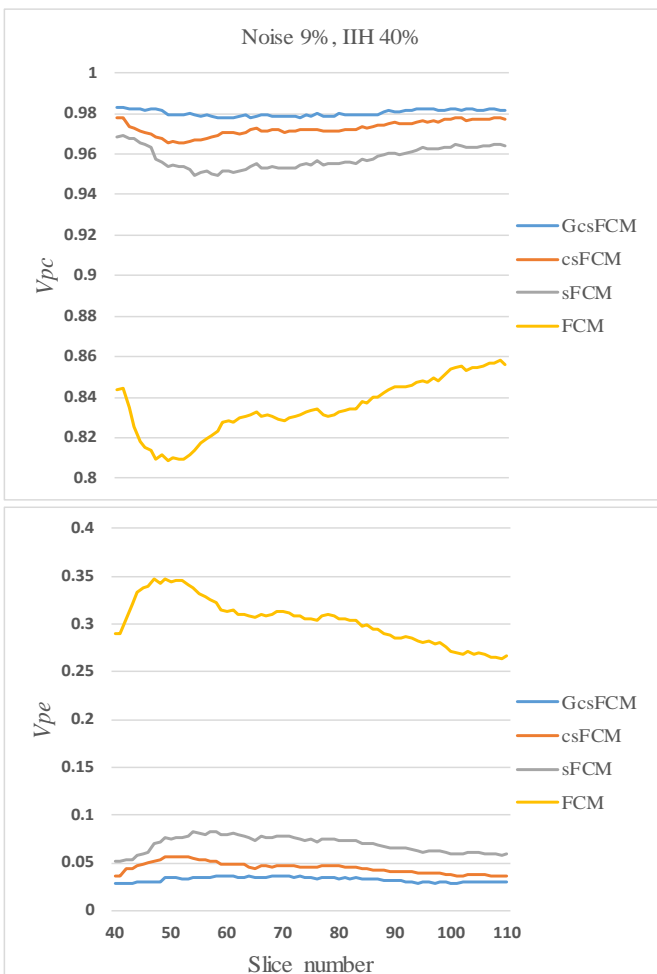

(a)

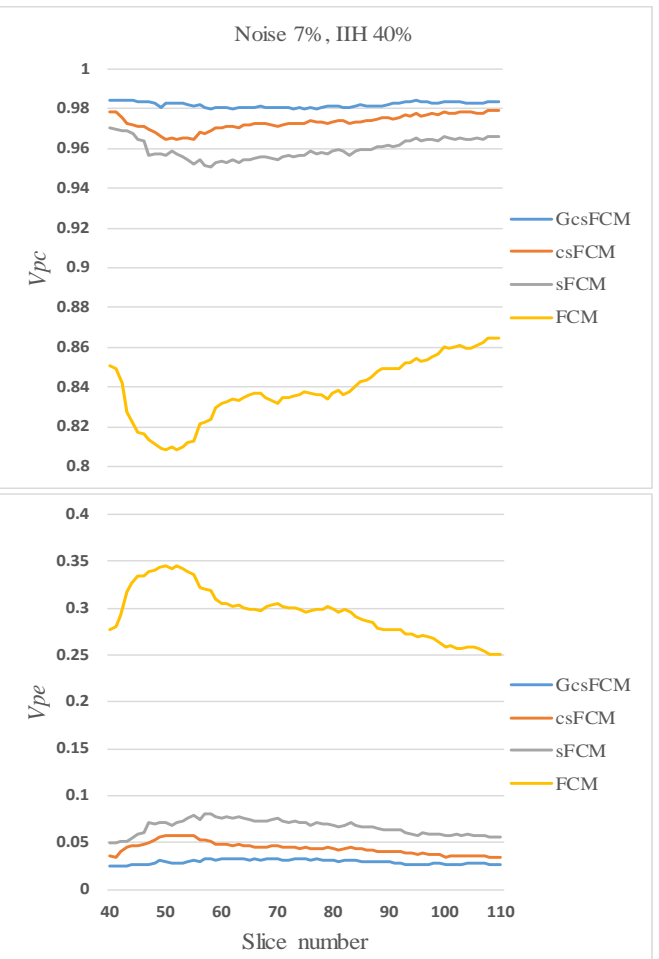

(c)

Fig. 4. Comparative values of $V_{p c}$ and $V_{p e}$ for the FCM, sFCM, csFCM and GcsFCM algorithms over 71 T1-weighted MRI brain images having 9\% noise and 40\% $\mathrm{IIH(a),} \mathrm{9 \%} \mathrm{noise} \mathrm{and} \mathrm{20 \%} \mathrm{IIH}(\mathbf{b}), 7 \%$ noise and $40 \% \operatorname{IIH(c)~and~} 7 \%$ noise and $20 \% \operatorname{IIH(d)}$. 
From Table 3, it can be seen that the proposed algorithm has better clustering effect. For the images with high noise and IIH, the average of $V_{p c}$ and $V_{p e}$ of the proposed GcsFCM method in the four image bodies are close to 1 and 0 , respectively. It shows that our algorithm has more accurate clustering results and less ambiguity than other algorithms. Furthermore, these results also prove the advantages to the competitive algorithms of the FCM, sFCM, and csFCM methods.

Table 3 shows the average for $V_{p c}$ and $V_{p e}$ of each image volume, but the specific assessed values for each image of each image volume are not clear, so the curves are plotted to display the values of $V_{p c}$ and $V_{p e}$. Fig. 4 shows the comparisons of the FCM, sFCM, csFCM, and GcsFCM algorithms for $V_{p c}$ and $V_{p e}$ of 71 different T1-weighted MRI brain images (slice 40-slice 110) with 9\% noise and 40\% IIH (Fig. 4(a)), 9\% noise and 20\% IIH (Fig. 4(b)), 7\% noise and 40\% IIH (Fig. 4(c)), and 7\% noise and 20\% IIH (Fig. 4(d)).

Fig. 4 gives an insight into the performance of the algorithm that the proposed algorithm has high value of Partition Coefficient $\left(V_{p c}\right)$ and low value of Partition Entropy $\left(V_{p e}\right)$, which shows that the proposed GcsFCM algorithm is performed consistently on all the images of the four data volumes. It also shows that it is superior to FCM, sFCM and csFCM algorithms.

\section{Conclusion}

In this paper, a Gamma correction conditional FCM clustering algorithm with spatial information (GcsFCM) is proposed. The advantage of the GcsFCM algorithm is that it still retains the details of the image and the edge information for MRI images with noise and IIH. The evidence from the experimental results is overwhelming. Our proposed algorithm has higher values of $V_{p c}$ and lower values of $V_{p e}$. High value of $V_{p c}$ implies better performance since it is calculated on the basis of membership values and high membership value for a single cluster implies better clustering. Similarly, low value of $V_{p e}$ implies better clustering. The experimental results are first presented in qualitatively and later in quantitatively along with critical discussions, which show that the proposed GcsFCM algorithm provides better segmentation results when compared to FCM, sFCM and csFCM algorithms.

\section{Acknowledgements}

This research is supported by the National Natural Science Foundation of China (61672259, 61602203), and Outstanding Young Talent Foundation of Jilin Province (20170520064JH). 

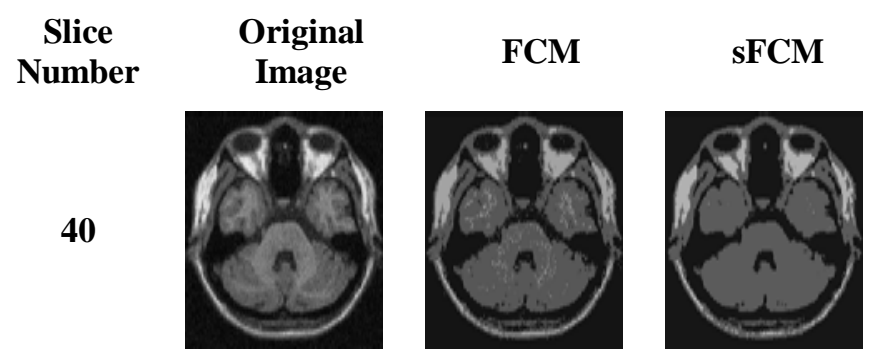

csFCM

GCsFCM

50
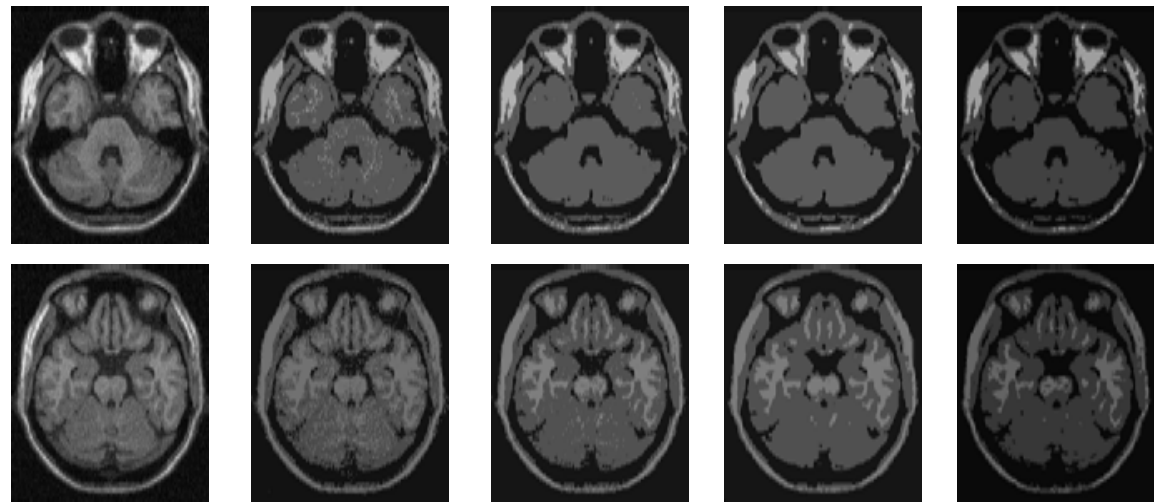

60
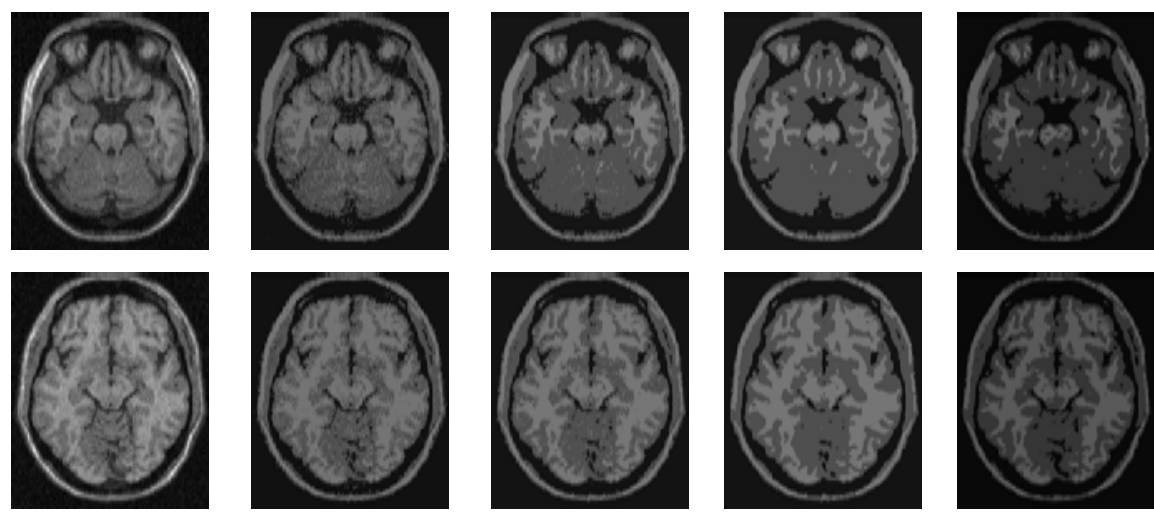

70
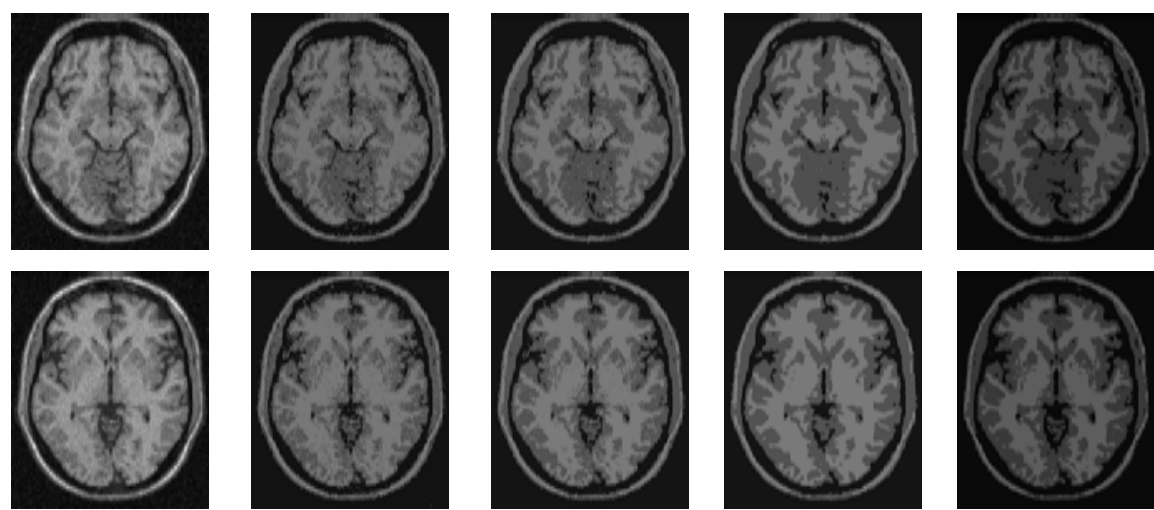

80
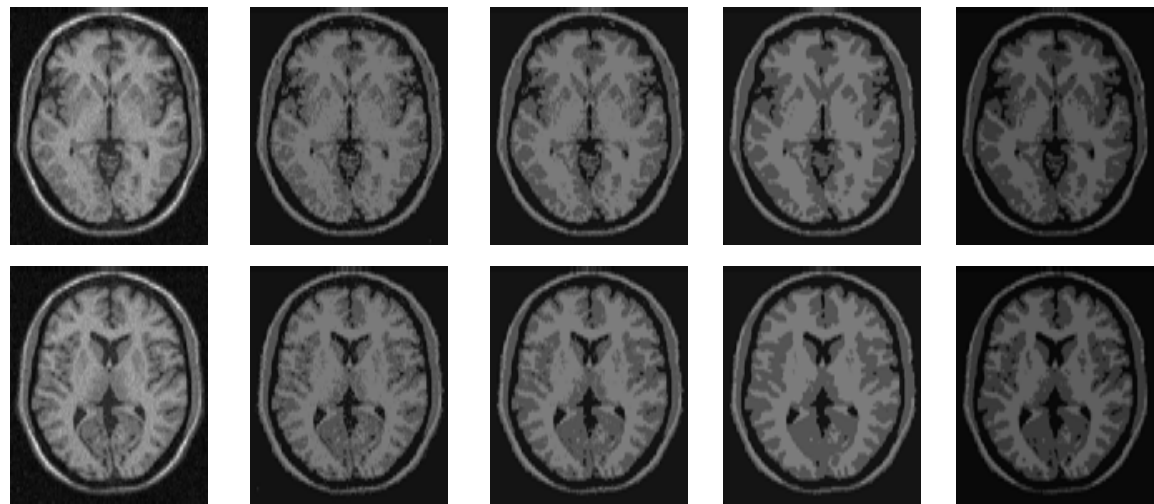

90
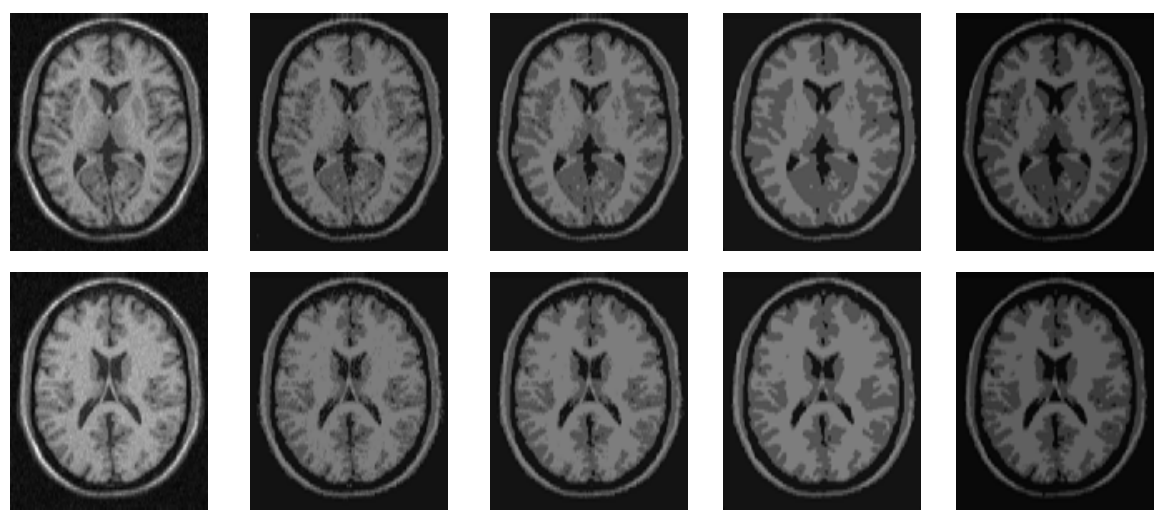

100
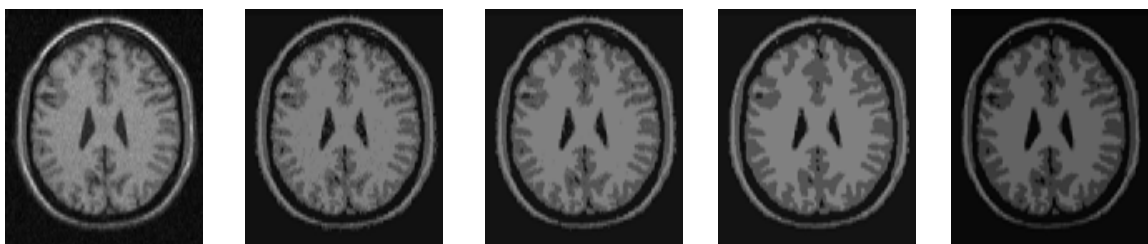

Fig. A1. Comparison results of FCM, sFCM, csFCM and our GcsFCM. Qualitative segmentation of different slice numbers $(40,50, \ldots, 100)$ of T1-weighted MRI brain images with $9 \%$ noise and 20\% inhomogeneity. 


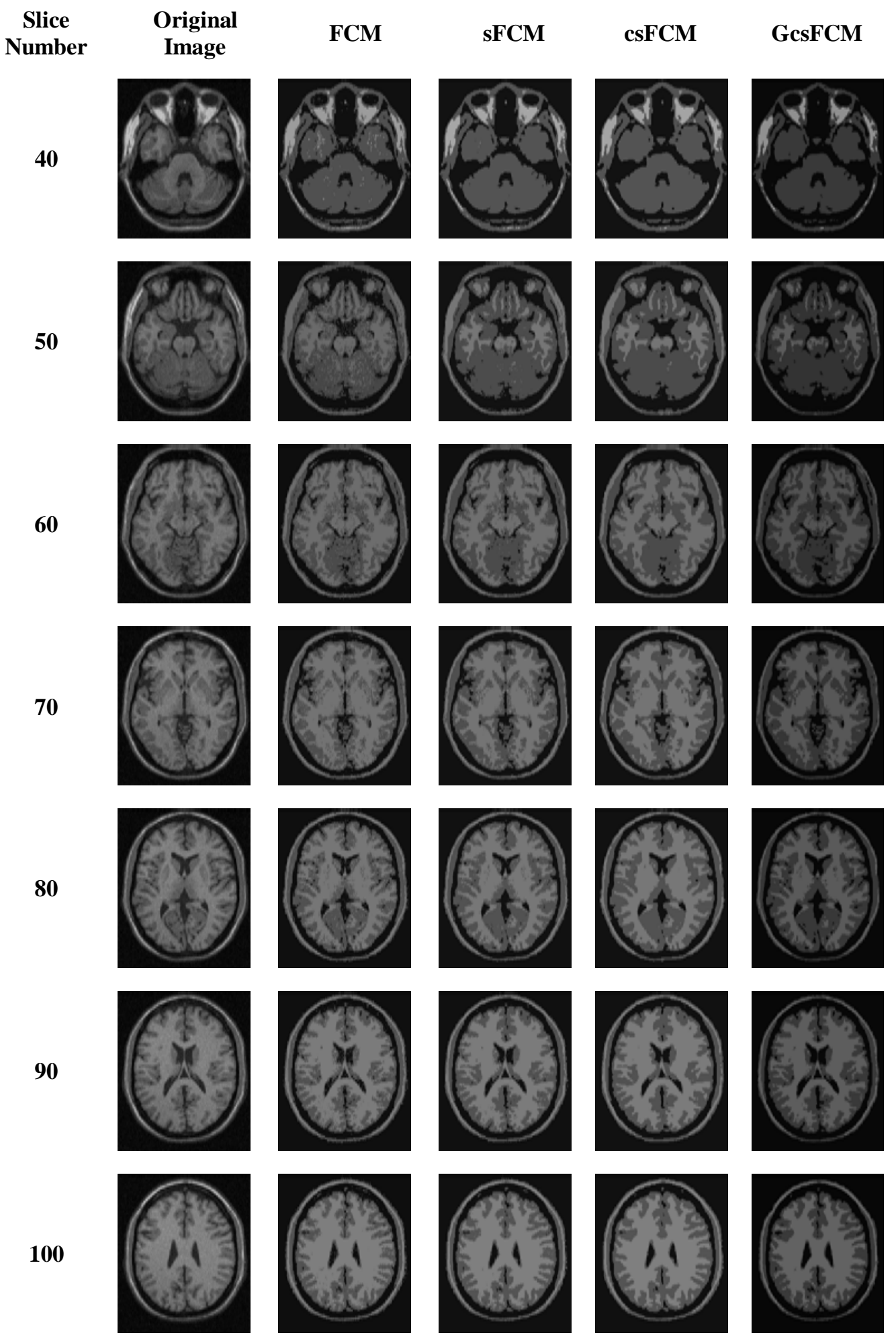

Fig. A2. Comparison results of FCM, sFCM, csFCM and our GcsFCM. Qualitative segmentation of different slice numbers $(40,50, \ldots, 100)$ of T1-weighted MRI brain images with $7 \%$ noise and $40 \%$ inhomogeneity. 


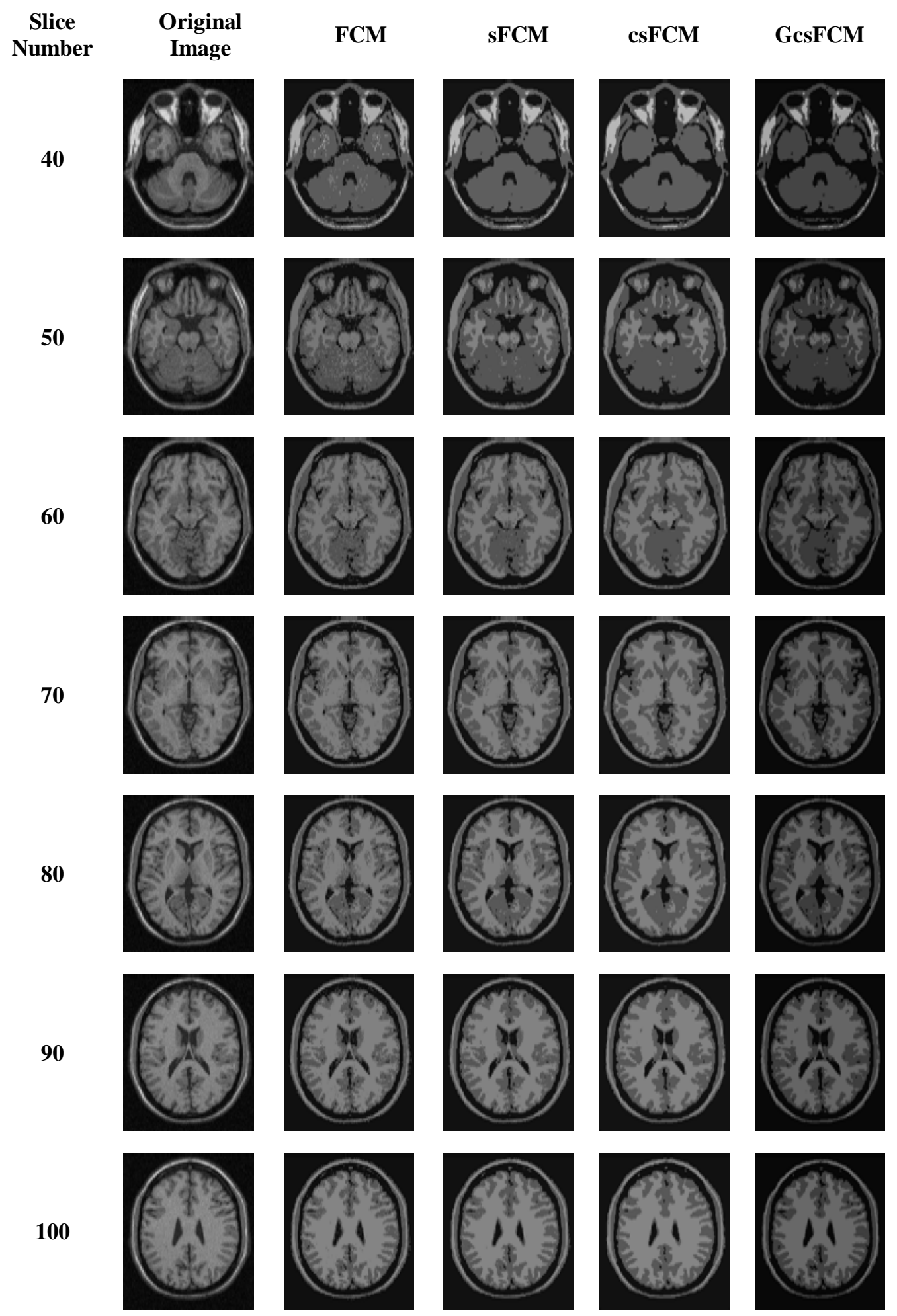

Fig. A3. Comparison results of FCM, sFCM, csFCM and our GcsFCM. Qualitative segmentation of different slice numbers $(40,50, \ldots, 100)$ of T1-weighted MRI brain images with $7 \%$ noise and $20 \%$ inhomogeneity. 


\section{References}

[1] M. A. Balafar, A. R. Ramli, M. I. Saripan and S. Mashohor. "Review of brain MRI image segmentation methods,” Artificial Intelligence Review, vol. 33, no. 3, pp. 261-274, January, 2010. Article (CrossRef Link).

[2] Kashif Hussain Memon and Dong-Ho Lee. "Generalised kernel weighted fuzzy C-means clustering algorithm with local information,” Fuzzy Sets and Systems, vol. 340, no. 1, pp. 91-108, June, 2018. Article (CrossRef Link).

[3] Hong Richang, Li Lei, Cai Junjie and Tao Dapeng. "Coherent Semantic-Visual Indexing for Large-Scale Image Retrieval in the Cloud,” IEEE Transactions on Image Processing, vol. 26, no. 9, pp. 4128-4138, September, 2017. Article (CrossRef Link).

[4] Peng Bo, Zhang Lei and Zhang David. "A survey of graph theoretical approaches to image segmentation,” Pattern Recognition, vol. 46, no. 3, pp. 1020-1038, March, 2013.

Article (CrossRef Link).

[5] Zhu Hongqing and Pan Xu. "Robust fuzzy clustering using nonsymmetric student's t, finite mixture model for MR image segmentation,” Neurocomputing, vol. 175(PA), pp. 500-514, January, 2016. Article (CrossRef Link).

[6] Qian Pengjiang, Zhao Kaifa, Jiang Yizhang, Su Kuanhao and Deng Zhaohong. "Knowledge-Leveraged Transfer Fuzzy C-Means for Texture Image Segmentation with Self-Adaptive Cluster Prototype Matching,” Knowledge-Based Systems, vol.130, pp. 33-50, August, 2017. Article (CrossRef Link).

[7] Jose L. Salmeron, Antonio Ruiz-Celma and Angel Mena. "Learning FCMs with multi-local and balanced memetic algorithms for forecasting industrial drying processes," Neurocomputing, vol. 232, pp. 52-57, April, 2017. Article (CrossRef Link).

[8] Chuxiong Sun and Xiangzhi Bai. "Cell segmentation based on spatial information improved intuitionistic fcm combined with FOPSO," in Proc. of IEEE Conf. on Image Processing, pp. 4457-4461, September 17-20, 2017. Article (CrossRef Link).

[9] Yong Yang, Ch Zheng and Pan Lin. "Fuzzy C-means clustering algorithm with a novel penalty term for image segmentation,” Opto-electronics Review, vol. 13, no. 4, pp. 309-315, January, 2005. Article (CrossRef Link).

[10] Songcan Chen and Daoqiang Zhang. "Robust image segmentation using FCM with spatial constraints based on new kernel-induced distance measure," IEEE Transactions on Systems Man \& Cybernetics Part B Cybernetics A Publication of the IEEE Systems Man \& Cybernetics Society, vol. 34, no. 4, pp. 1907-1916, August, 2004. Article (CrossRef Link).

[11] L. Szilagyi, Z. Benyo, S. M. Szilágyi and H. S. Adam. "MR brain image segmentation using an enhanced fuzzy c-means algorithm," in Proc. of IEEE conf. on Engineering in Medicine and Biology Society, vol. 1, pp. 724-726, September 17-21, 2003. Article (CrossRef Link).

[12] K. S. Chuang, H. L. Tzeng, S. Chen and Jay Wu. "Fuzzy c-means clustering with spatial information for image segmentation,” computerized medical imaging and graphics, vol. 30, no. 1, pp. 9-15, January, 2006. Article (CrossRef Link).

[13] Ji Zexuan, Xia Yong, Chen Qiang and Sun Quansen. "Fuzzy c-means clustering with weighted image patch for image segmentation,” Applied soft computing, vol. 12, no. 6, pp. 1659-1667, June, 2012. Article (CrossRef Link).

[14] Bai Xiangzhi, Chen Zhiguo, Zhang Yu and Liu Zhaoying. "Infrared ship target segmentation based on spatial information improved fcm," IEEE transactions on cybernetics, vol. 46, no. 12, pp. 3259-3271, December, 2016. Article (CrossRef Link).

[15] M. N. Ahmed, S. M. Yamany, N. Mohamed, A. A. Farag and T. Moriarty. "A modified fuzzy c-means algorithm for bias field estimation and segmentation of MRI data,” IEEE transactions on medical imaging, vol. 21, no. 3, pp. 193-199, March, 2002. Article (CrossRef Link).

[16] Ji Zexuan, Liu Jinyao, Cao Guo and Sun Quansen. "Robust spatially constrained fuzzy c-means algorithm for brain MR image segmentation,” Pattern Recognition, vol. 47, no. 7, pp. 2454-2466, July, 2014. Article (CrossRef Link). 
[17] Sudip Kumar Adhikari, Jamuna Kanta Sing, Dipak Kumar Basu and Mita Nasipuri. “Conditional spatial fuzzy C-means clustering algorithm for segmentation of MRI images,” Applied soft computing, vol. 34, pp. 758-769, September, 2015. Article (CrossRef Link).

[18] Xiao LiangJiang, Qiang Wang, Biao He, Shao JieChen and Bai LinLi. "Robust level set image segmentation algorithm using local correntropy-based fuzzy c-means clustering with spatial constraints,” Neurocomputing, vol. 207, pp. 22-35, September, 2016. Article (CrossRef Link).

[19] Richang Hong, Luming Zhang, Chao Zhang and Roger Zimmermann. "Flickr Circles: Aesthetic Tendency Discovery by Multi-View Regularized Topic Modeling," IEEE Transactions on Multimedia, vol. 18, no. 8, pp. 1555-1567, August, 2016. Article (CrossRef Link).

[20] Richang Hong, Jinhui Tang, Hung-Khoon Tan, Chong-Wah Ngo and Shuicheng Yan Tat-Seng Chua. "Beyond search: Event-driven summarization for web videos," Acm Transactions on Multimedia Computing Communications \& Applications, vol. 7, no. 4, November, 2011. Article (CrossRef Link).

[21] Richang Hong, Luming Zhang and Dacheng Tao. "Unified Photo Enhancement by Discovering Aesthetic Communities from Flickr,” IEEE Transactions on Image Processing, vol. 25, no. 3, pp. 1124-1135, March, 2016. Article (CrossRef Link).

[22] Kin Gwn Lore, Ade dotun Akintayo and Soumik Sarka. "LLNet: A deep autoencoder approach to natural low-light image enhancement,” Pattern Recognition, vol. 61, pp. 650-662, January, 2016. Article (CrossRef Link).

[23] Y. S. Zhou, K. J. Yi, M. Mahjouri-Samani and W. Xiong. "Image contrast enhancement in field-emission scanning electron microscopy of single-walled carbon nanotubes,” Applied Surface Science, vol. 255, no. 7, pp. 4341-4346, January, 2009. Article (CrossRef Link).

[24] Jing RuiTang and Nor Ashidi Mat Isa. "Bi-histogram equalization using modified histogram bins," Applied Soft Computing, vol. 55, pp. 31-43, June, 2017. Article (CrossRef Link).

[25] Amandeep Kaur and Chandan Singh. "Contrast enhancement for cephalometric images using wavelet-based modified adaptive histogram equalization,” Applied Soft Computing, vol. 51, pp. 180-191, February, 2017. Article (CrossRef Link).

[26] Akash Gandhamal, SanjayTalbar, Suhas Gajre and Ahmad Fadzil M.Hani. "Local gray level S-curve transformation-A generalized contrast enhancement technique for medical images," Computers in biology and medicine, vol. 83, pp. 120-133, April, 2017. Article (CrossRef Link).

[27] Wei Xian, Yuanxiang Li, Hao Shen and Weidong Xiang. "Joint learning sparsifying linear transformation for low-resolution image synthesis and recognition,” Pattern Recognition, vol. 66, pp. 412-424, June, 2017. Article (CrossRef Link).

[28] Jianji Wang, Nanning Zheng, Yuehu Liu and Gang Zhou. "Parameter analysis of fractal image compression and its applications in image sharpening and smoothing," Signal Processing: Image Communication, vol. 28, no. 6, pp. 681-687, July, 2013. Article (CrossRef Link).

[29] I. Frosio, G. Ferrigno and N. A. Borghese. "Enhancing digital cephalic radiography with mixture models and local gamma correction," IEEE transactions on medical imaging, vol. 25, no. 1, pp. 113-121, January, 2006. Article (CrossRef Link).

[30] Richang Hong, Zhenzhen Hu, Ruxin Wang, Meng Wang and Dacheng Tao. "Multi-View Object Retrieval via Multi-Scale Topic Models,” IEEE Transactions on Image Processing, vol. 25, no. 12, pp. 5814-5827, December, 2016. Article (CrossRef Link).

[31] C. Cocosco. "Brainweb: Online interface to a 3D MRI simulated brain database,” NeuroImage. vol. 5, pp. 425, 1997. Article (CrossRef Link).

[32] J. C. Bezdek. “Cluster validity with fuzzy sets,” Journal of Cybernetics, vol. 3, no. 3, pp. 58-73, 1973. Article (CrossRef Link).

[33] Shi Jiao, Lei Yu, Zhou Ying and Gong Maoguo. "Enhanced rough-fuzzy c-means algorithm with strict rough sets properties,” Applied Soft Computing, vol. 46, pp. 827-850, September, 2016. Article (CrossRef Link).

[34] J. C. Bezdek. "Mathematical models for systematics and taxonomy," in Proc. of eighth international conference on numerical taxonomy, vol. 3, pp. 143-166, January, 1975. Article (CrossRef Link). 

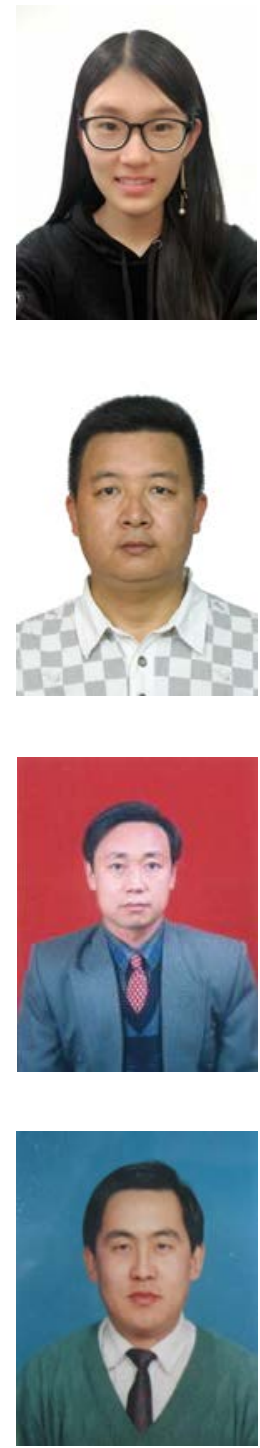

Yang Liu is a student of the college of computer science and technology, Jilin University. She is a member of Key Laboratory of Symbolic Computation and Knowledge Engineering of Ministry of Education. Her research interests include image processing and pattern recognition.

Haipeng Chen, was born in 1978, is a professor of the college of computer science and technology, Jilin University. He is a member of Key Laboratory of Symbolic Computation and Knowledge Engineering of Ministry of Education. His research interests include image processing, pattern recognition and multimedia information security.

Xuanjing Shen, was born in 1958, is a professor of the college of computer science and technology, Jilin University. He is a member of Key Laboratory of Symbolic Computation and Knowledge Engineering of Ministry of Education. His research interests include multimedia technology, image processing, intelligent measurement system and optical-electronic hybrid system.

Yongping Huang is an associate professor of the college of computer science and technology, Jilin University. He is a member of Key Laboratory of Symbolic Computation and Knowledge Engineering of Ministry of Education. His research interests include Embedded Software and Information Physics Fusion System. 